\title{
Action on smoking — opportunities for the dental team
}

\author{
R. G. Watt, ' N. W. Johnson, ${ }^{2}$ and K. A. A. S. Warnakulasuriya, ${ }^{3}$
}

\begin{abstract}
In 1998, the UK government published a White Paper outlining a comprehensive range of measures to reduce smoking rates across the population. In the same year a detailed overview of the evidence base for smoking cessation activities within the NHS was published. Both these documents provide useful information for health professionals interested in developing their roles in smoking cessation and prevention. An increased risk for the development of oral malignacies and a susceptibility for the breakdown of periodontal tissues are the most significant effects of smoking on the mouth. This paper aims to highlight how dentists and their team members can become actively involved in efforts to reduce smoking. Opportunities at both a clinical and public health level are considered.
\end{abstract}

Smoking remains a significant public Shealth problem worldwide. Each year in the UK 120,000 premature deaths are caused by tobacco smoking. It is the largest single preventable cause of mortality and morbidity, and costs the NHS $£ 1700$ million a year. ${ }^{1}$ In recognition of the impact of smoking, the Government has recently published a comprehensive anti-smoking strategy that outlines a range of complementary actions aimed at reducing smoking rates across the population. ${ }^{2}$ This paper aims to highlight the vital role dentists and their team members can play both at a clinical and public health level in meeting the goals set out in this strategy.

\section{Epidemiology of smoking}

Tobacco use peaked in the early 1960s and steadily declined until very recently. At present there are around 13 million adult

\footnotetext{
${ }^{*}$ Senior Lecturer, Department of Epidemiology and Public Health, Royal Free and University College London Medical School, University College London, 1-19 Torrington Place, London WC1E 6BT; ${ }^{2}$ Reader, ${ }^{3}$ Professor, Department of Oral and Maxiollofacial Medicine and Pathology, The Guy's, Kings and St Thomas' Schools of Medicine, Dentistry and Biomedical Sciences, GKT Dental Institute, Caldecot Road, London SE5 9RW

${ }^{\star}$ Correspondence to: $R$. G. Watt

email:r.watt@ucl.ac.uk

REFEREED PAPER

Received 05.07.99; Accepted 03.08.99

(C) British Dental Journal 2000; 189: 357-360
}

In brief

- Smoking is a major public health problem in the UK.

- Evidence-based guidelines provide clear guidance to health professionals on effective ways of helping patients to stop smoking.

- Dentists and their teams have an opportunity to become involved in smoking cessation activities.

- A range of public health measures are also required to support clinician's efforts against smoking.

smokers in the UK, $28 \%$ of the population. ${ }^{1}$ Rates of smoking, however, vary considerably across the population and are a major cause of inequalities in life expectancy between socio-economic groups. In 1996, $12 \%$ of men in professional occupations smoked compared with $40 \%$ of men in unskilled manual jobs. ${ }^{3}$ Increasingly smoking is becoming restricted to disadvantaged groups in society.

A major cause for concern is the recent increase in smoking among young people, especially teenage girls. In $19888 \%$ of 11-15-year-olds in England were regular smokers. By 1996 the proportion had risen to $13 \%{ }^{4}$ Eighty-two per cent of adult smokers start smoking during adolescence. Many of these smokers will continue to smoke for decades, addicted to nicotine, unable to give up and therefore at increasing risk of serious illness and premature death.

\section{Policy context}

Action on smoking is now firmly on the Government policy agenda. In December 1998 Smoking Kills, A White Paper on Tobacco was published. ${ }^{2}$ This document sets out a comprehensive agenda for action on smoking and fits in with the subsequent public health strategy, Our Healthier Nation. ${ }^{5}$ Government policy is now concentrating on reducing health inequalities through joint action on the underlying determinants of disease in society. This is a radically different approach to the health policy pursued by the previous government. Rather than focusing attention on changing individual lifestyles, policies are now recognising the importance of modifying the social environment to promote health and reduce health inequalities.

The new tobacco strategy has set three objectives:

- To reduce smoking among children and young people

- To help adults, especially the most disadvantaged to give up smoking

- To offer particular help to pregnant women who smoke.

These objectives are to be met through a comprehensive range of measures as outlined in Table 1.

The government has allocated $\mathfrak{E 5 0}$ million to a 3-year media campaign to persuade smokers to quit and non smokers, especially young people, not to start. The campaign was launched in December 1999 and will use a range of media activities. Part of the campaign will involve developing information packs that health professionals, including dentists' can use to inform their patients about the dangers of smoking and encourage cessation attempts.

The success of the range of measures outlined in the White paper will be judged against three new targets that have been set: 


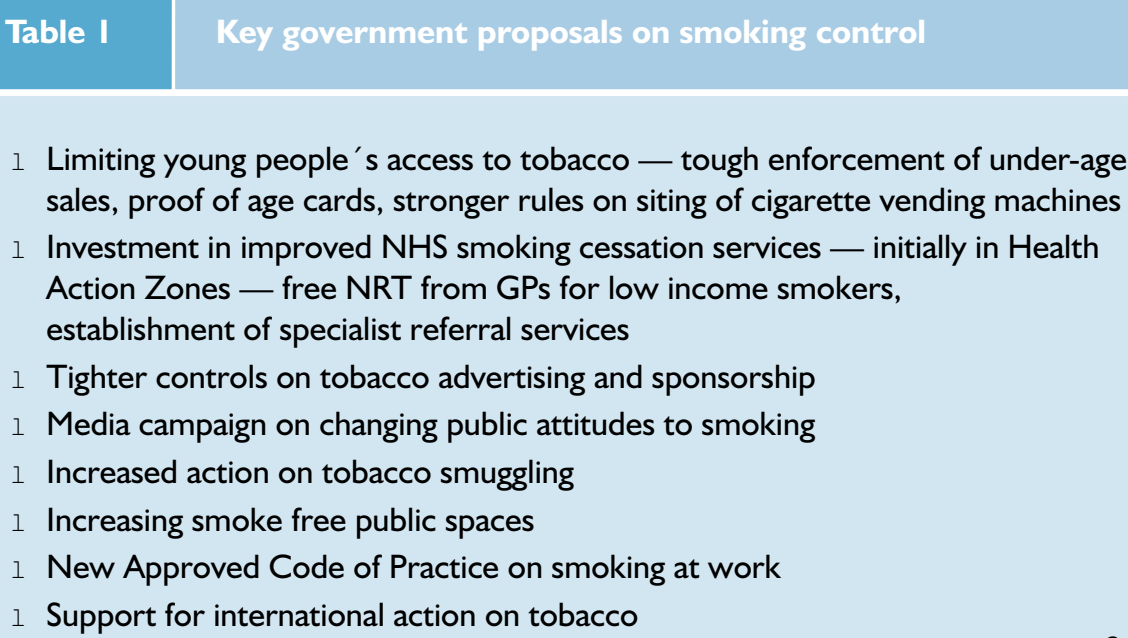

1 Limiting young people's access to tobacco - tough enforcement of under-age sales, proof of age cards, stronger rules on siting of cigarette vending machines

1 Investment in improved NHS smoking cessation services — initially in Health Action Zones - free NRT from GPs for low income smokers, establishment of specialist referral services

1 Tighter controls on tobacco advertising and sponsorship

Media campaign on changing public attitudes to smoking

Increased action on tobacco smuggling

Increasing smoke free public spaces

New Approved Code of Practice on smoking at work

Support for international action on tobacco

Source: Smoking Kills, $1998 .^{2}$

3. To reduce the percentage of women who smoke during pregnancy from $23 \%$ to $15 \%$ by the year 2010 ; with a fall to $18 \%$ by the year 2005 .

This national strategy will be supported by action at a local level through activities developed in Health Improvement Programmes and Health Action Zones. Resources are being made available to develop action plans across the NHS and especially in primary care settings.

\section{Smoking and oral health - opportunities for action}

There are important links between smoking and oral health that provide a unique opportunity for action by the dental team. Tobacco use has many well recognised pathological effects on the oral tissues. Oral cancers and precancers, increased severity and extent of periodontal diseases and poor wound healing are the most significant effects of smoking on the mouth. ${ }^{6,7}$

Smoking remains the major risk factor for oral cancer and heavy smokers carry a high relative risk (seven times more than non smokers: several times more in smokers who also consume alcohol heavily). ${ }^{8}$ The attributable risk of smoking for oral cancer is estimated between $75-90 \%$. There is clear evidence to suggest that by successfully giving up smoking after a 10 -year-period this increased risk falls to a level close to that of a person who has never smoked. ${ }^{9}$ This information could be helpful in motivating patients to stop smoking.

The early effects of smoking are often clearly evident on the facial and oral tissues. These changes can be visible to patients and are reversible on successful cessation. They can therefore be used as a marker of the impact of smoking on the body and in motivational counselling. Halitosis and tooth staining are also common concerns of quitting. ${ }^{10}$

The new tobacco strategy has placed particular emphasis upon decreasing smoking among young people and pregnant woman. ${ }^{2}$ Both these groups routinely use dental services on a regular basis. Dental professionals therefore have a direct opportunity to influence these groups during clinical care and at follow up appointments. This is a unique position of great potential influence.

\section{Evidence-based approach}

Smoking cessation delivered in primary care has been shown to be very effective. Recently published effectiveness reviews have systematically evaluated the evidence and identified an international consensus on smoking cessation recommendations. ${ }^{11}$ Very brief advice lasting less than 3 minutes given by a health professional will help an additional $2 \%$ of smokers to successfully stop smoking. With more intensive support lasting up to 10 minutes, plus nicotine replacement therapy (NRT) an additional $6 \%$ of smokers will quit. ${ }^{11}$ These quit rates may seem rather insignificant but when translated into a population estimate between 63,000 and 190,000 people may quit smoking each year in the UK if all general dental practitioners routinely offered smoking cessation advice to those seeking dental care through an opportunistic approach.

\section{Smoking cessation within dental practice settings}

Although relatively few studies have been published, smoking cessation programmes conducted through dental practices report cessation rates comparable to studies in smokers and can be used as motivations for other primary care settings. ${ }^{12,13}$ However in spite of the apparent appropriateness of the dental practice as a setting for smoking cessation/prevention and the positive attitude many dentists have towards their involvement in this activity, at present relatively few general dental practitioners routinely provide smoking advice for their patients. ${ }^{14,15}$ Reported barriers to providing smoking advice include time and cost pressures, concerns regarding the effectiveness of interventions, inadequate training and a lack of appropriate resources. ${ }^{13,15-18}$

In an attempt to encourage dentists to become more involved a smoking cessation protocol has been developed to give advice and support in the most effective manner in the dental practice. ${ }^{19}$ The 4 A's model is a simple and quick means of identifying smokers who want to quit and how best to support them in their quit attempt. ${ }^{20}$ To be effective smoking cessation advice needs to be tailored to the smokers' circumstance and experience (Fig. 1).

Ask - All patients should have their smoking status checked at the start of each course of treatment. A simple and quick system should be devised to record smoking details in patient's clinical notes. This should assess whether the person smokes and their motivation to stop. These notes need to be kept up-to-date and should be included in a standard medical history. Suitable questions are on the BDA's medical history proforma.

Advise - All smokers should be advised of the value of quitting. Most people are fully aware of the health dangers of smoking in relation to lung cancer and heart disease. The public is less aware however of the links between smoking and oral diseases. ${ }^{21}$ The dental team are in a unique position to high- 


\section{PRACTICE $\underline{\text { smoking cessation }}$}

Fig. I Smoking cessation protocol

\section{Ask}

All patients should have their smoking status checked

Advise

All smokers should be advised on the value of quitting

Assist

For those smokers who want to stop appropriate support should be offered

\section{Arrange}

Monitoring, follow up and referral should be arranged

light this and to identify other motivations for stopping such as the social and hygiene consequences of smoking. The early and reversible effects of smoking on the oral tissues can be used as strong motivators to quit. Stained teeth, halitosis and soft tissue changes in the mouth may be especially pertinent to young people. All smokers will have their own good reasons for stopping. Advice given should therefore be personalised, clear and strong.

Assist - for those smokers who want to stop, appropriate support should be offered. This should include:

- Advice on setting a definite quit date

- Review past experiences of quitting determine what happened, identify what helped and hindered progress

- Plan ahead to predict problems and devise ways of dealing with these

- Enlist the support of the patient's family and friends - a critical element for success

- The value of NRT should be discussed

- Stress the free support and advice available from Quitline (0800 002200). This year a new NHS smoking helpline has been launched by the Department of Health (0800 1690169).

Arrange - As in any other clinical situation monitoring progress is an essential element of the intervention. Ideally patients should be seen 1-2 weeks after their quit date to assess their progress. This may well fit with a subsequent dental appointment for on going treatment or a visit to the hygienist. Congratulate patients who are succeeding - praise can motivate patients and help them maintain their new behaviour. Those patients who are smoking again also need encouragement and support. A patronizing 'finger wagging' approach helps no one. Instead it is important to establish what happened and the lessons that can be drawn from the experience. Sensitive questioning can help patients reflect and understand the reasons for smoking again. Most smokers make several attempts at quitting over many months and even years before they are successful. ${ }^{22}$ These attempts should be considered as learning opportunities not failures.

For some smokers who have made many unsuccessful attempts at quitting or who have experienced severe withdrawals from nicotine, specialist cessation services are available for more intensive help. Specialist cessation services are being established across the country. Up-to-date details are available from the Quitline (0800 002200) or NHS smoking helpline (0800 1690169).

The routine incorporation of smoking cessation advice and support within dental practices offers several potential benefits to both staff and patients (Table 2).

\section{Nicotine replacement therapy}

One of the most difficult problems in quitting is dealing with the inevitable cravings for nicotine. A course of nicotine replacement therapy (NRT) helps with this and when used correctly doubles the cessation rate. ${ }^{11}$ NRT is not a miracle cure for nicotine addiction but provides nicotine without any harmful smoke. A variety of products are now available on the market (gum, patch, nasal spray, inhalator and microtabs), all of which have similar success rates. The choice of product largely depends on personal preference. To promote use among low income smokers, a 1-week free supply of NRT is now available within Health Action Zones across England. Later this year the scheme will be extended to other parts of the country.

Various complementary therapies may be helpful in assisting some people in their attempts at quitting although the research base for these therapies is not as extensive as NRT.

\section{Team approach}

To be effective smoking cessation requires a team approach within dental practices. ${ }^{13}$ Each member of the dental team can play an important contribution. Dentists may take the lead in assessing smoking status and raising the issue onto the agenda with their patients. Other team members such as hygienists and dental nurses may provide the more detailed assistance to patients. The Health Development Agency is currently developing a training resource for dental hygiene tutors which aims to develop hygiene students skills in smoking cessation. Reception staff also have an important role to play. A selection of health education materials are available for use in dental practices. These

cessation advice and support

1 Promote general health and oral health of patients

Improve the effectiveness of clinical interventions eg periodontal surgery

Develop team roles and responsibilities

Opportunity for joint working with other health professionals

Improve standing and status of practice as a health promoting setting 


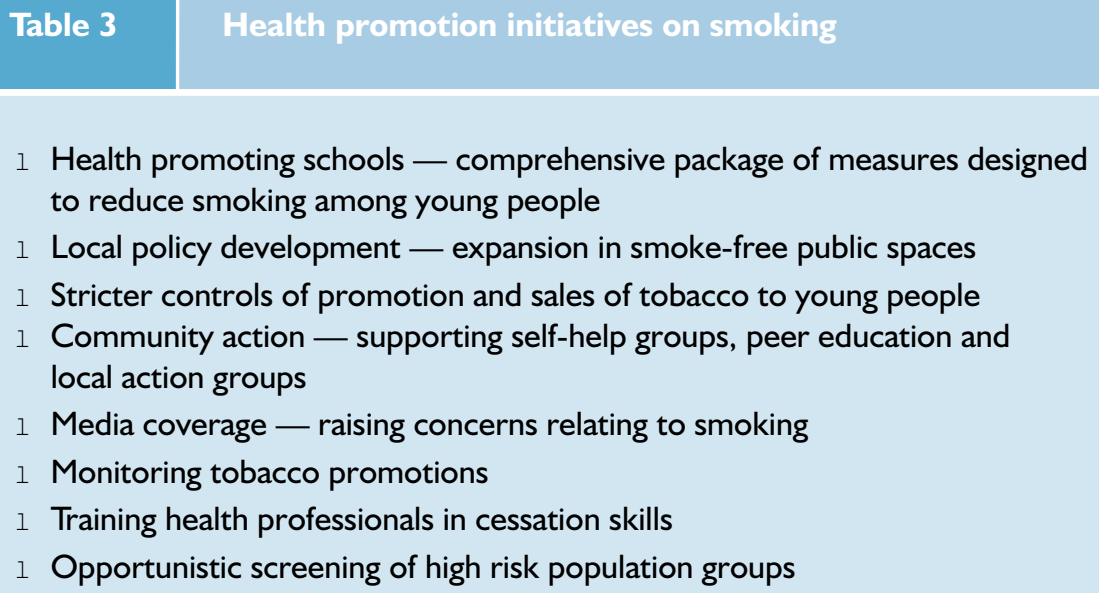

1 Health promoting schools - comprehensive package of measures designed to reduce smoking among young people

1 Local policy development - expansion in smoke-free public spaces

1 Stricter controls of promotion and sales of tobacco to young people

1 Community action - supporting self-help groups, peer education and local action groups

Media coverage - raising concerns relating to smoking

Monitoring tobacco promotions

Training health professionals in cessation skills

Opportunistic screening of high risk population groups

are available through local health promotion units.

\section{Dental public health measures}

Although action at a clinical level is important and is to be encouraged, public health measures are also required to reduce smoking at a population level. Dental public health practitioners and community dental teams have a particular role to play in this area. However general dental practitioners, through groups such as the British Dental Association, can also have an influence as forces for change.

The White Paper on smoking outlines a range of measures which dental public health and community dental staff could play an important role in, especially in relation to the proposals for young people (Table 3). Effective public health action is needed to address the underlying reasons why people start and continue to smoke. The barriers that prevent successful quitting also need to be tackled. Action needs to be co-ordinated across sectors and agencies well beyond the health service. Local government, the retail and commercial sector, education authorities and community organisations all need to work colloboratively together. It is also essential that the local population are fully involved in any actions undertaken. Using a range of complementary actions is essential to increase the effectiveness of health education interventions at a clinical level. ${ }^{23}$

At present one of the reasons given by dental practitioners for not routinely offering smoking cessation advice is a lack of time and the costs incurred. ${ }^{14,17}$ Although it can be argued that through capitation payments GDPs are already receiving some reimbursement for this service a dedicated fee for smoking cessation advice would surely encourage greater provision. Professional groups such as the BDA have a lobby- ing role to play with the Department of Health for a revision in practitioners fees to include cessation advice.

\section{Conclusion}

Smoking kills 120,000 people each year in the UK. The government has published a comprehensive anti-smoking strategy that has been broadly welcomed by the health professions and anti smoking groups. A range of actions are now being developed and implemented to reduce smoking across the population but especially among young people, pregnant women and adults in disadvantaged settings. The dental profession have an important contribution to play in combating the smoking epidemic. Evidence-based guidelines implemented in primary dental care settings would have a significant impact on smoking rates. A successful cessation programme in dental practices has demonstrated the benefits of this approach. ${ }^{13}$ Effective smoking cessation not only benefits the health and well-being of patients but also improves outcomes of other treatments. The dental team are in a unique and strong position to help many smokers to stop through simple, quick and personalised advice and support.

The authors are grateful to Dr Ann McNeill at the Health Education Authority for her helpful comments on this manuscript.

1 Callum C. The UK smoking epidemic: Deaths in 1995. London: Health Education Authority, 1998.

2 Smoking Kills: A White Paper on Tobacco. London: The Stationery Office, 1998.

3 Thomas M, Walker A, Wilmot A, Bennet N. Office for National Statistics. Living in Britain: results from the 1996 General Household Survey. London: The Stationery Office, 1998.

4 Jarvis L. Smoking among secondary school children in 1996: England. Office of National Statistics. London: The Stationery Office, 1997.

5 Saving Lives: Our Healthier Nation. London: The Stationery Office, 1999.
6 Legarth J, Reibel J. EU Working Group on Tobacco and Oral Health. Oral Dis 1998; 4: 48-67.

7 Johnson N W, Bain C. Tobacco and oral disease. Br Dent J 2000; 4: 200-206.

8 Cancer Research Campaign. Cancersheet; Oral Cancer - UK. London: Cancer Research Campaign, 2000.

9 Franco E, Kowalski L, Oliveria B et al. Risk factors for oral cancer in Brazil: a case control study. Int J Cancer 1989; 43: 992-1000.

10 Owen L, Bolling K. Tracking teenage smoking. London: Health Education Authority, 1995.

11 Raw M, McNeill A, West R. Smoking cessation guidelines for health professionals. A guide to effective smoking cessation interventions for the health care system. Thorax 1998; Suppl. 5: 1-38.

12 Cohen S J, Stookey G K, Katz B P, Drook C A Christen A G. Helping smokers quit: a randomised controlled trial with private practice dentists. JADA 1989; 118: 41-45.

13 Smith S E, Warnakulasuriya K A A S, Feyerabend C, Belcher M, Cooper D J, Johnson $\mathrm{N}$ W. A smoking cessation programme conducted through dental practices in the UK. Br Dent J 1998; 185: 299-303.

14 John J H, Yudkin P, Murphy M, Ziebland S, Fowler G H. Smoking cessation interventions for dental patients-attitudes and practices of dentists in the Oxford region. Br Dent J 1997; 183: 359-364.

15 McCann M, Macpherson L M D, Binnie V. et al. A survey of Scottish primary care dental practitioners' oral cancer-related practices and training requirements. Com Dent Health 2000; 17: 24-30.

16 Chestnutt I G, Binnie V I. Smoking cessation counselling-a role for the dental profession? $\mathrm{Br}$ Dent J 1995; 179: 411-415.

17 Warnakulasuriya K A A S, Johnson N W. Dentists and oral cancer prevention in the UK: opinions, attitudes and practices of screening for mucosal lesions and to counselling patients on tobacco and alcohol use: baseline data from 1991. Oral Dis 1999; 5: 10-14.

18 Allard R H B. Tobacco and oral health: attitudes and opinions of EU dentists; a report of the EU working group on tobacco and oral health. Int Dent J 2000; 50: 99-102.

19 Watt R, Robinson M. Helping smokers to stopa guide for the dental team. London: Health Education Authority, 1999.

20 Glynn T J, Manley M W. How to help your patients stop smoking. A National Cancer Institute Manual for Physicians. Washington: US Department of Health and Human Services, 1989.

21 Warnakulasuriya K, Harris C, Scarrott D. et al. An alarming lack of public awareness towards oral cancer. Br Dent J 1999; 187: 319-322.

22 Prochaska J O, Goldstein M.G. Process of smoking cessation: Implications for clinicians. Clin Chest Med 1991; 42: 727-775.

23 Sprod A, Anderson R, Treasure E. Effective oral health promotion. Literature review. Technical report no. 20 Cardiff: Health Promotion Wales, 1996. 